University of Wollongong

Research Online

Faculty of Engineering and Information

Faculty of Engineering and Information

Sciences - Papers: Part B

Sciences

2019

Ensemble Neural Network Method for Wind Speed Forecasting

Binbin Yong

Fei Qiao

Chen Wang

Jun Shen

University of Wollongong, jshen@uow.edu.au

Yongqiang Wei

See next page for additional authors

Follow this and additional works at: https://ro.uow.edu.au/eispapers1

Part of the Engineering Commons, and the Science and Technology Studies Commons

Research Online is the open access institutional repository for the University of Wollongong. For further information contact the UOW Library: research-pubs@uow.edu.au 


\title{
Ensemble Neural Network Method for Wind Speed Forecasting
}

\author{
Abstract \\ Wind power generation has gradually developed into an important approach of energy supply. Meanwhile, \\ due to the difficulty of electricity storage, wind power is greatly affected by the real-time wind speed in \\ wind fields. Generally, wind speed has the characteristics of nonlinear, irregular, and non-stationary, which \\ make accurate wind speed forecasting a difficult problem. Recent studies have shown that ensemble \\ forecasting approaches combining different sub-models is an efficient way to solve the problem. \\ Therefore, in this article, two single models are ensembled for wind speed forecasting. Meanwhile, four \\ data pre-processing hybrid models are combined with the reliability weights. The proposed ensemble \\ approaches are simulated on the real wind speed data in the Longdong area of Loess Plateau in China \\ from 2007 to 2015, the experimental results indicate that the ensemble approaches outperform individual \\ models and other hybrid models with different pre-processing methods.

\section{Keywords} \\ speed, wind, method, forecasting, network, ensemble, neural \\ Disciplines \\ Engineering | Science and Technology Studies

\section{Publication Details} \\ Yong, B., Qiao, F., Wang, C., Shen, J., Wei, Y. \& Zhou, Q. (2019). Ensemble Neural Network Method for Wind \\ Speed Forecasting. 2019 IEEE International Workshop on Signal Processing Systems (pp. 31-36). United \\ States: IEEE.

\section{Authors} \\ Binbin Yong, Fei Qiao, Chen Wang, Jun Shen, Yongqiang Wei, and Qingguo Zhou
}




\section{Ensemble Neural Network Method for Wind Speed Forecasting}

\author{
Binbin Yong \\ School of Information Science \\ and Engineering, Lanzhou \\ University \\ School of Physical Science and \\ Technology, Lanzhou University \\ Lanzhou, China \\ yongbb@1zu.edu.cn
}

\author{
Fei Qiao \\ Dept. of Electronic Engineering, \\ Tsinghua University \\ Beijing, China \\ qiaofei@tsinghua.edu.cn
}

\author{
Chen Wang \\ School of Information Science \\ and Engineering, Lanzhou \\ University \\ Lanzhou, China \\ chwang2017@1zu.edu.cn
}

\author{
Jun Shen \\ School of Computing and \\ Information Technology, \\ University of Wollongong, \\ Dept. of EE and CS, Research \\ Lab of Electronics, \\ Massachusetts Institute of \\ Technology, Cambridge \\ NSW, Australia \\ jshen@uow.edu.au
}

\author{
Yongqiang Wei \\ School of Information Science \\ and Engineering, Lanzhou \\ University \\ Lanzhou, China \\ weiyq18@1zu.edu.cn
}

\author{
Qingguo Zhou \\ School of Information Science \\ and Engineering, Lanzhou \\ University \\ Lanzhou, China \\ zhouqg@1zu.edu.cn
}

\begin{abstract}
Wind power generation has gradually developed into an important approach of energy supply. Meanwhile, due to the difficulty of electricity storage, wind power is greatly affected by the real-time wind speed in wind fields. Generally, wind speed has the characteristics of nonlinear, irregular, and non-stationary, which make accurate wind speed forecasting a difficult problem. Recent studies have shown that ensemble forecasting approaches combining different sub-models is an efficient way to solve the problem. Therefore, in this article, two single models are ensembled for wind speed forecasting. Meanwhile, four data pre-processing hybrid models are combined with the reliability weights. The proposed ensemble approaches are simulated on the real wind speed data in the Longdong area of Loess Plateau in China from 2007 to 2015, the experimental results indicate that the ensemble approaches outperform individual models and other hybrid models with different pre-processing methods.
\end{abstract}

Key words: wind power generation; wind speed forecasting; ensemble forecasting approaches; hybrid models

\section{Introduction}

At present, energy and environment problems have become the main problems of global sustainable development. Meanwhile, with the maturity of wind power generation technology, it is widely used for its non-pollution and renewability. According to statistics [1], in 2015, China accounted for $28.4 \%$ of the world's wind capacity with 305,000 kilowatts of power generation, while the world's new wind capacity increased by $22 \%$ to 63013 megawatts. The cumulative annual growth rate had reached $17 \%$ in 2015. Due to the good market environment, China has a tremendous future in wind energy market.
Generally, the power of wind-generated electricity is mainly depended on the wind conditions in the wind farms. Hence, wind conditions forecasting has great significance for wind power conversion in wind farm management. In order to forecast the wind speeds more accurately, many researches have been conducted. There are many prediction models, such as traditional statistical and machine learning models [2-4]. Literature review shows that if time series data include both linear and non-linear models, only statistical models or machine learning models can not accurately predict wind speed. Therefore, no method or model can play a good role in all cases $[5,6]$. Meanwhile, many multi-step forecasting models that combine the traditional linear statistical model with neural network algorithms are also proposed based on traditional combination method, pre-processing method, parameter optimization technology, data post-processing algorithm [7-13], etc. A hybrid model proposed by Wang et al. [7] achieved better prediction performance by capturing different sequential patterns. Guo et al. [11] studied a modified hybrid method based on seasonal adjustment and autoregressive moving average (ARIMA) model to predict wind speed. Hu et al. [13] designed an ensembled EEMD-SVM approach for wind speed forecasting.

Recently, ensemble models are widely researched for wind speed forecasting. Most of these studies focus on how to reasonably obtain the weight of each single prediction method, and effectively improve the prediction accuracy [14, 15]. However, because of the nonlinearity and unstable characteristics, a single model may not be sufficient for wind 
speed forecasting. The prediction results of traditional machine learning approaches are determined by the stability of wind speeds. On the other hand, the traditional weighted combination model has little effect on noise suppression, and the prediction results may be inaccurate [15].

In order to deal with the chaos and instability of wind speed series, back propagation neural network (BP) is often used for wind speed forecasting for its nonlinearity. Meanwhile, support vector machine (SVM) model, as a type of model based on structural risk minimization, is also a better choice for small samples forecasting, such as wind speed forecasting. Therefore, in this paper, BP and SVM are adopted as the base forecasting models of ensemble models. Meanwhile, four hybrid models based on set empirical mode decomposition (EEMD) and wavelet transform (WT) are established respectively. Overall, ensemble models including EEMD-BP, EEMD-SVM, WT-BP and WT-SVM are designed to deal with the wind speed forecasting issue in the next year. The models WT-BP, WTSVM, EEMD-BP and EEMD-SVM are combined with no negative constraint theory (CM-NNCT) and the chaos particles warm optimization algorithm for combination method (CPSOCM) methods to establish comprehensive prediction approaches, which are compared to the regression models of BP and SVM.

The rest of this paper is organized as follows. The wind speed dataset and the proposed ensemble approaches are described in Section 2. In Section 3, the proposed methods are applied to forecast the wind speed in the wind speed dataset, and the results are discussed. In Section 4, we summarize the whole paper.

\section{Dataset and ensemble approaches}

The wind speed dataset used in this paper is collected from the Loess Plateau in Longdong area in Gansu province, China. The features of the daily wind speed data set are explored by using Jonckheere-Terpstra $(\mathrm{J}-\mathrm{T})$ test. The simulation process is conducted to check the effectiveness of proposed models.

\subsection{Wind speed dataset}

The study area is located in the northwest of Longdong Loess Plateau with an elevation of 1200-2089 meters. Geomorphology consists of ridges, hills, valleys and ravines. There are 106 mountains, 400 hills, 17364 rivers and 527 gullies in the northwest of Longdong Loess Plateau. The total land area of the town is 9236 square kilometers, with a population of 347,000 , of which $93.7 \%$ is agricultural population. It meanwhile has average rainfall and sunshine of about 300 millimeters and 108 days respectively, which resulted in serious soil erosion and environmental degradation. It is an intersection point centered on mountainous areas, and the special geographical environment and backward economic development make it important for national strategy.

With the requirement of new energy construction and poverty alleviation, the industrial structure is being adjusted and new energy resources are being developed in the surrounding areas. In June 2011, the construction of a MW wind power generation base began in the surrounding area, and a total installed capacity of about $100 \mathrm{MW}$ has been planned. Construction of 110 kilovolt booster station will convert the power of Tianshuibao substation into the state grid. Since its commissioning in late June 2012, the equipment still work well. In October 2012, it generated more than 1,000 kilovolts electricity $(\mathrm{kWh})$.

Therefore, in order to optimize network management and provide more wind power energy, more accurate wind speed forecasting models are needed. In this article, the wind speed dataset in the Huan region in Longdong area of the Loess Plateau are collected from 1 January 2007 to 31 December 2015 for wind speed forecasting experiments. Fig. 1 show the details of the area providing wind speed dataset. Meanwhile, the wind speed data used in this paper is also presented.

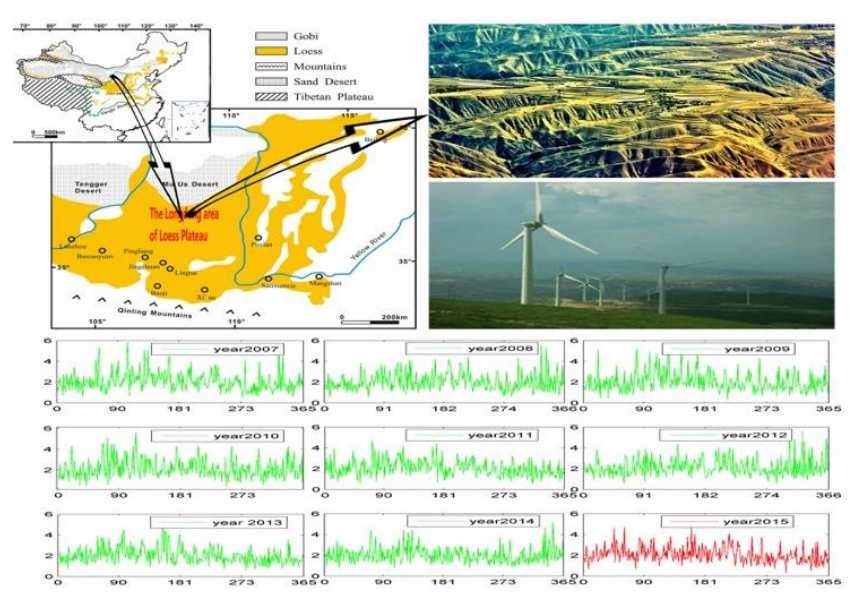

Fig. 1. Daily average wind speed curves for the Huan region from 2007 to 2015.

In our design, the wind speed data from 1/1/2007 to $31 / 12 / 2014$ is utilized to train the forecasting models, and the dataset from $1 / 1 / 2015$ to $31 / 12 / 2015$ is adopted to test the models. It is necessary that the J-T test is performed to check whether the test dataset has significant difference with the training dataset or not.

\subsection{Data pre-processing technique used in the ensemble models}

As discussed above, the wind speed data has nonlinear, irregular, and highly-noisy characteristics because it is determined by meteorological factors and other stochastic factors. Meanwhile, wind speed forecasting with noisy data will suffer from large forecasting errors. Hence, we need to remove the noise in the wind speed data firstly. In this paper, WT and ensemble EEMD [16] pre-processing techniques are utilized to eliminate the noisy data. Then, BP and SVM are adopted for forecasting. Therefore, four types of hybrid models, including WT-BP, WT-SVM, EEMD-BP and EEMD-SVM are tested.

\subsection{The ensemble models}

Time series forecasting, as a key technology in predicting almost all social and economic activities, has been widely researched in the past few decades. Previous researches mainly 
focused on the single forecasting models, such as ARIMA, BP and SVM models. ARIMA is a traditional linear regression model, which assumes the time series a random sequence and utilizes a certain mathematical model to approximate the time series, so as to predict future sequence data by the mathematical model. However, ARIMA is suitable for simple time series data with linear characteristics such as economic growth forecast, and it is inappropriate for complex time series such as wind speed prediction. In this case, BP and SVM are often adopted to model the complex time series with nonlinear characteristics. BP is often designed with multilayer perceptron (MLP) and optimized by back propagation algorithm. MLP consists of many hidden nodes, which are in fact equal to nonlinear functions. Hence, BP can capture the inherent nonlinear law of time series very well, and it can be used as a basic method for wind speed forecasting. SVM is another commonly used method for small samples forecasting. Unlike the empirical risk minimization (ERM) strategy used in BP, SVM adopts the structural risk minimization (SRM) strategy to find an optimal hyperplane to separate samples, which was later developed for regression forecasting applications and also called as support vector regression (SVR). In fact, SVM utilizes a nonlinear kernel function to map the original data into a new space to solve the nonlinear problem, which can also be seen as a type of neural network. Hence, SVM can also be tried for nonlinear wind speed forecasting.

BP and SVM models train learning machines based on ERM and SRM respectively, both of which can handle nonlinear wind speed forecasting problem. In fact, they work well in different situations. In order to ensemble the advantages of each model, we combine BP and SVM by different methods in this paper. In fact, ensemble approaches combining two or more single models have been widely researched [15]. The proposed combination models can be categorized as traditional combined approaches, data preprocessing method, parameter optimization technique and data post-processing algorithms [15, 17-19] etc. In this article, we apply new ensemble methods for wind forecasting, which are different from traditional combined method. BP and SVM are used as the base models of combined models, and four hybrid models based on WT-BP, WT-SVM, EEMD-BP and EEMD-SVM data pre-processing methods are ensembled based on the proposed CM-NNCT and the CPSOCM methods.

\subsection{Experimentation design and evaluation metrics}

In our experiments, we collected the wind speed dataset in the Huan region to test the forecasting accuracy of the proposed ensemble models, and the specific experimental process is presented as following steps:

Firstly: Perform the J-T test for the dataset, by which we judge whether the wind speed dataset (training samples and test samples) have significant differences between each other or not.

Secondly: We apply two single models, including BP neural network and SVM, into wind speed forecasting, and the forecasting errors are discussed.

Thirdly: Four hybrid forecasting models based on different pre-processing techniques including WT-BP neural network,
WT-SVM, EEMD-BP neural network and EEMD-SVM are utilized to predict the average wind speed, and the forecasting results are analyzed.

Fourthly: The forecasting results of BP neural network, SVM, WT-BP, WT-SVM, EEMD-BP, EEMD-SVM are compared, and the CM-NNCT and CPSO-CM are adopted to find the weights of each component model in the ensemble models. The forecasting result of these ensemble models are discussed.

To test the forecasting results, four metrics including mean absolute error (MAE), mean square error (MSE), and mean absolute percentage error (MAPE) are adopted as below:

$$
\begin{gathered}
\text { MAE }=\frac{1}{n} \sum_{i=1}^{n}\left|x_{i}-\hat{x}_{i}\right| \\
\mathrm{MSE}=\frac{1}{n} \sum_{i=1}^{n}\left|x_{i}-\hat{x}_{i}\right|^{2} \\
\mathrm{MAPE}=\frac{1}{n} \sum_{i=1}^{n}\left|\frac{x_{i}-\hat{x}_{i}}{x_{i}}\right|
\end{gathered}
$$

where $x_{i}$ and $\hat{x}_{i}$ denote the $i$ th real value and the $i$ th forecasting value. $n$ represents the number of samples.

\section{Simulation results}

In this section, we use the J-T test method to explore the features of the wind speed dataset. The results are shown in Table 1. It gives the differences of observed J-T test value and mean J-T test value. The significant level value is set as 0.05 , then the $p$-value of J-T statistic is $p=0.24>0.05$, which means that the wind speed values have inherent law and can be used for forecasting.

Table 1 The results of J-T test.

\begin{tabular}{lll}
\hline J-T test & & Wind speed \\
\cline { 1 - 1 } Grouping Variable.cs & & 9 \\
$\mathrm{~N}$ & & 3287 \\
Observed J-T statistic & & 2437581.0 \\
Mean J-T statistic & & 2400970.5 \\
Std. deviation of J-T statistic & & 31187.144 \\
Std. J-T statistic & 1.174 \\
Asymp. Sig. (2.tailed) & 0.240 \\
\hline
\end{tabular}

\subsection{The forecasting results of single BP and SVM models}

In this subsection, two single regression models, BP neural network and SVM, are utilized for wind speed forecasting experiments, and the results are shown in Fig. 2. We can see that the forecasting results of SVM and BP are not close to those observed for the peaks of the series, illustrating that these prediction models do not resemble the observed series. The MAE, MSE and MAPE metrics are given in Table 2.
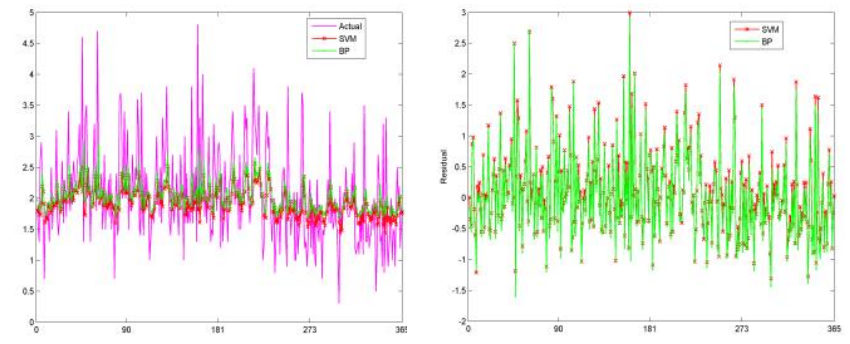
Fig. 2. The forecasting results (left) and errors (right) of SVM and BP neural network.

The forecasting interval in this study is set as one year, and the forecasting result shows that the MAPE by BP neural network and SVM are $31.02 \%$ and $29.37 \%$ respectively, which are considered desirable. As shown in Table 2, three metrics of SVM models are all smaller than the BP model. It indicates that the SVM outperforms BP model in our wind speed dataset.

Although the results of the SVM are smaller, forecasting results by these two models are both far from the observed values. Hence, we need to ensemble BP neural network and SVM to achieve better forecasting results.

\subsection{The experimental results of WT-BP, WT-SVM, EEMD- $B P$ and EEMD-SVM models}

In this subsection, four hybrid wind speed forecasting models including WT-BP neural network, WT-SVM, EEMDBP neural network and EEMD-SVM are tested.

The wind speed data are decomposed into several layers via the wavelet transform, then the WT-BP neural network model and WT-SVM model are established using the lowfrequency signal of every layer for prediction. Table 2 shows that the WT-BP model and WT-SVM model selected in this process outperform the conventional BP model and SVM model despite the improvement is not significant, and the reason may be that the WT de-noising method is prone to be influenced by the threshold.

In order to overcome the inadequacy of the WT method, the EEMD method is adopted to preprocess the original data, which is decomposed into several independent IMFs where IMFn denotes the lowest frequency band and IMF1 represents the highest frequency signals. In this paper, the IMF1 and IMFn are neglected since they have little features of the original data. The original data is de-noised by EEMD, then the BP neural network and SVM model (EEMD-BP and EEMD-SVM) are established using the de-noised data to predict the wind speed in 2015 respectively. The prediction results and residuals of WT-BP, WT-SVM, EEMD-BP and EEMD-SVM are shown in Fig. 3. We can conclude that the developed EEMD-BP and EEMD-SVM regression models perform better than the BP, SVM, WT-BP and WT-SVM models.
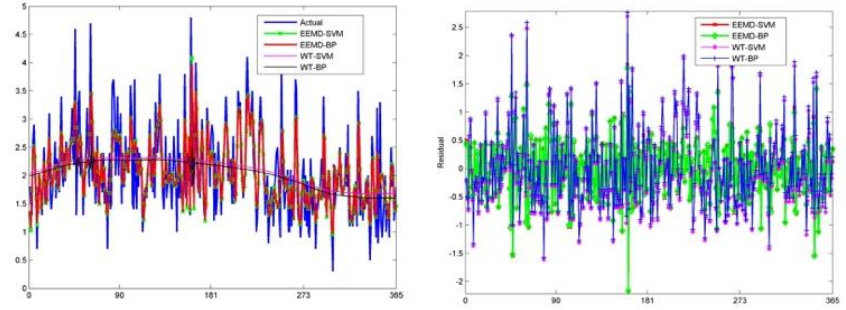

Fig. 3. The prediction results and residuals of WT-BP, WTSVM, EEMD-BP and EEMD-SVM.

The MSE, MAE and MAPE are also shown in Table 2, which show that EEMD-BP and EEMD-SVM have smaller forecasting errors compared to BP, SVM, WT-BP and WT-
SVM models. In fact, the MAPE of EEMD-BP and EEMDSVM significantly decreased about $7 \%$, while the MAPE of WT-BP and WT-SVM decreased less than 1\%, compared with forecasting results of SVM and BP. It means that prediction accuracies of EEMD-BP and EEMD-SVM are higher than WTBP and WT-SVM, while the prediction accuracy of EEMD-BP is similar to EEMD-SVM.

\subsection{The forecasting results of $\mathrm{CM}-\mathrm{NNCT}$ and $\mathrm{CPSO}-\mathrm{CM}$ models}
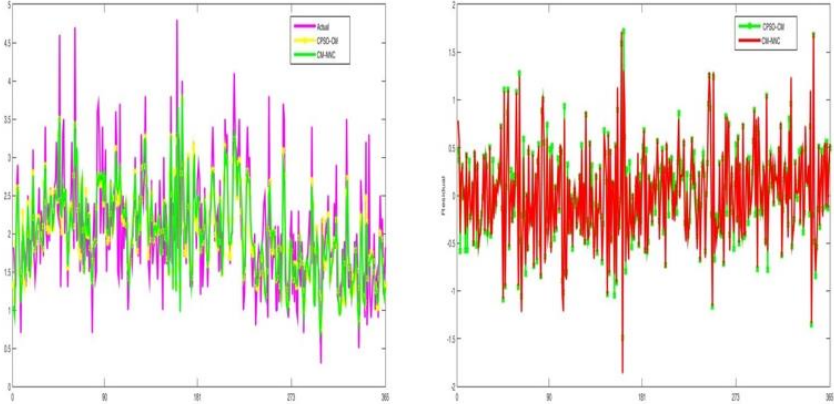

Fig. 4. The forecasting results and residuals of CM-NNCT and $\mathrm{CPSO}-\mathrm{CM}$

In this subsection, MSE metric is calculated to test the wind speed forecasting results for different predict models. The forecasting results and errors of CM-NNCT and CPSO-CM models are presented in Fig. 4. The MSE, MAE and MAPE metrics are shown in Table 2.

The comparison results between CM-NNCT and CPSO$\mathrm{CM}$ showed that the CM-NNCT and CPSO-CM models yielded similar results, while the MAE, MSE and MAPE of CPSO-CM are all smaller than CM-NNCT, which means that the forecast precision of CPSO-CM is higher than the CMNNCT model.

The experimental results indicate that the CM-NNCT and CPSO-CM models perform better than the BP, SVM, WT-BP, WT-SVM, EEMD-BP and EEMD-SVM. Compared with EEMD-BP and EEMD-SVM, the MAPE of CM-NNCT decreased about $1.37 \%$ and $1.52 \%$, and the MAPE of CPSOCM significantly decreased about $1.7 \%$ and $1.85 \%$. We can conclude from Fig. 5 that the MAE, MSE and MAPE metrics by CPSO-CM and CM-NNCT methods are smaller than BP, SVM, WT-BP, WT-SVM, EEMD-BP and EEMD-SVM.

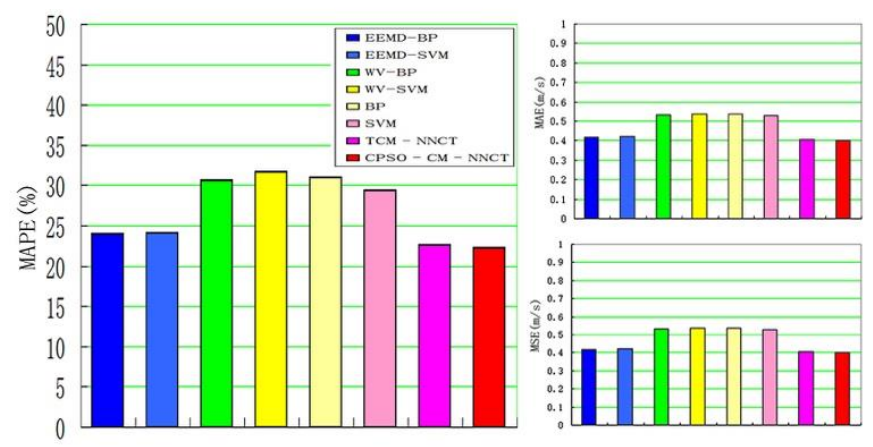


Fig. 5. Three metrics of BP, SVM, WT-BP, WT-SVM, EEMD-BP, EEMD-SVM. CPSO-CM and CM-NNCT

In Table 2 we also give the MSE, MAE and MAPE of CPSO-CM and CM-NNCT. When comparing with BP, SVM, WT-BP, WT-SVM, EEMD-BP and EEMD-SVM, we can see that CPSO-CM and CM-NNCT models have the higher forecasting precision than single models and hybrid models in this research instances.

Table 2 The MSE, MAE and MAPE metrics of CPSO-CM and CM-NNCT compared with BP, SVM, WT-BP, WT-SVM, EEMD-BP and EEMD-SVM.

\begin{tabular}{|c|c|c|c|c|}
\hline $\begin{array}{l}\text { Evaluation } \\
\text { criteria }\end{array}$ & CPSO-CM & CM-NNCT & EEMD-SVM & EEMD-BP \\
\hline $\operatorname{MSE}(\mathrm{m} / \mathrm{s})$ & 0.2514 & 0.2603 & 0.2828 & 0.2778 \\
\hline $\operatorname{MAE}(\mathrm{m} / \mathrm{s})$ & 0.4008 & 0.4062 & 0.4223 & 0.4196 \\
\hline MAPE $(\%)$ & 22.24 & 22.57 & 24.09 & 23.94 \\
\hline $\begin{array}{l}\text { Evaluation } \\
\text { criteria }\end{array}$ & WT-BP & WT-SVM & BP & SVM \\
\hline $\operatorname{MSE}(\mathrm{m} / \mathrm{s})$ & 0.4838 & 0.4821 & 0.4956 & 0.5011 \\
\hline $\operatorname{MAE}(\mathrm{m} / \mathrm{s})$ & 0.531 & 0.5374 & 0.5354 & 0.5289 \\
\hline MAPE (\%) & 30.59 & 31.65 & 31.02 & 29.37 \\
\hline
\end{tabular}

\section{Conclusions}

Wind speed forecasting is still a great challenge in the field of time series forecasting. We have proposed and tested BP and SVM models and four hybrid models (WT-BP, WT-SVM, EEMD-BP and EEMD-SVM) based on different preprocessing methods for wind speed forecasting. Experimental results illustrate that these forecasting models need to be ensembled to get better forecasting results. Next, the BP, SVM, WT-BP, WT-SVM, EEMD-BP, EEMD-SVM are combined by the CM-NNCT and CPSO-CM models. The MAPE of these ensemble models are $22.57 \%$ and $22.24 \%$. The experimental results illustrated that the ensemble approaches have high prediction accuracy. Moreover, the comparisons between the conventional single forecasting models (BP neural network and SVM) and the hybrid models (WT-BP, WT-SVM, EEMD-BP, EEMD-SVM) show that ensemble models outperform single forecasting models.

\section{References}

[1] http://www.cehome.com/news/20160314/210615.shtml

[2] R.G. Kavasseri, K. Seetharaman, Day-ahead wind speed forecasting using f-ARIMA models, Renewable Energy 34 (2009) 1388-1393.

[3] Li G, Shi J. On comparing three artificial neural networks for wind speed forecasting. Appl Energy 2010,87,2313-2320.

[4] Morales JM, Minguez R, Conejo AJ. A methodology to generate statistically dependent wind speed scenarios. Appl Energy 2010;87(3):843-855.

[5] C. Chatfield, what is the 'best' method of forecasting? Journal of Applied Statistics 15 (1998) 19-39.
[6] G. Zhang, Time series forecasting using a hybrid ARIMA and neural network model, Neuro computing 50 (2003) 159175.

[7] J. Wang, W. Zhang, J. Wang, T. Han, L. Kong, A novel hybrid approach for wind speed prediction, Information Sciences (2014), doi: http://dx.doi.org/10.1016/j.ins.2014.02.159.

[8] Zhongyue Su, Jianzhou Wang, Haiyan Lu, Ge Zhao. A new hybrid model optimized by an intelligent optimization algorithm for wind speed forecasting. IEnergy Convers Manag 2014, 85: 443-452.

[9] Zhao W, Wang J, Lu H. Combining forecasts of electricity consumption in China with time-varying weights updated by a high-order Markov chain model. Omega2014,45: 80-91.

[10] Bouzgou H, Benoudjit N. Multiple architecture system for wind speed prediction. Appl Energy2011,88:2463-2471.

[11] Zhenhai Guo, Yao Dong, Jianzhou Wang, Haiyan Lu, The Forecasting Procedure for Long-Term Wind Speed in the ZhangYe Area, Mathematical Problems in Engineering, Volume 2010, Article ID 684742, 17 pages doi:10.1155/2010/684742.

[12] Lei C, Ran L. Short-term wind speed forecasting model for wind farm based on wavelet decomposition. In: Proceeding soft the third international conference on electric utility Deregulation and Restructuring and Power Technologies (DRPT); 2008.P.2525-2529.

[13] Jianming Hu, Jianzhou Wang, Guowei Zeng, A hybrid forecasting approach applied to wind speed time series, Renewable Energy 60 (2013) 185-194.

[14] Ma Y,Tang X. Multi-objective optimal combination forecast model. Stat Res 1997(4)5-8.

[15] Ling Xiao, JianzhouWang, YaoDong, JieWu. Combined forecasting models for wind energy forecasting: A case study in China. Renewable and Sustainable Energy Reviews, 2015 (44):271-288

[16] Wu Z, Huang NE. Ensemble empirical mode decomposition: a noise-assisted data analysis method, centre for ocean-land-atmosphere studies. Tech Rep 2004,193(51).

[17] Homayounia Seyed Mahdi,Tang Sai Hong, Motlagh Omid. A genetic algorithm for optimization of integrated scheduling of cranes, vehicles, and storage platforms at automated container terminals. J Comput Appl Math 2014(270):545-56.

[18] Chao Ren, Ning An, Jianzhou Wang, Lian Li, Bin Hu, Duo Shang, Optimal parameters selection for BP neural network based on particle swarm optimization: A case study of wind speed forecasting, Knowledge-Based Systems 56 (2014) 226239.

[19] Zhenhai Guo, Weigang Zhao, Haiyan Lu, Jianzhou Wang, Multi-step forecasting for wind speed using a modified EMDbased artificial neural network model, Renewable Energy 37 (2012) 241-249. 